\title{
A case of melatonin-induced biochemical flare in a patient with primary sclerosing cholangitis with features of autoimmune hepatitis
}

\author{
Maciej Gonciarz ${ }^{1}$, Aldona Mularczyk ${ }^{1}$, Dawid Szkudłapski ${ }^{1}$, Stanislaw J. Konturek ${ }^{2}$, Peter C. Konturek ${ }^{2}$ \\ 1'Department of Gastroenterology, St Barbara's Main District Hospital, Sosnowiec, Poland \\ ${ }^{2}$ Department of Physiology, Jagiellonian University Medical College, Krakow, Poland
}

Gastroenterology Rev 2019; 14 (2): 157-159

DOI: https://doi.org/10.5114/pg.2019.85901

Address for correspondence: Prof. Maciej Gonciarz MD, PhD, Department of Gastroenterology, St Barbara's Main District Hospital, 1 Plac Medykow, 40-200 Sosnowiec, Poland, e-mail: m_gonciarz@poczta.fm

Melatonin ( $\mathrm{N}$-acetyl-5-methoxy-triptamine) is the major product of the pineal gland; however, it has also been identified in a large number of extrapineal organs including the gastrointestinal tract, cornea, bone marrow, lymphocytes, etc. [1]. Melatonin has well-known anti-oxidative, oncostatic, and anti-aging properties and numerous experimental and clinical data have established its beneficial effects [2-4]. Melatonin exerts some of its physiological effects in immune cells through two G-protein-coupled specific membrane receptors: MT1 (CAMP signalling pathway) and MT2 (cGMP signalling pathway). There is now evidence that melatonin possesses important immunoenhancing properties, mostly underlined in immunodepressed situations [3], and it may counteract the immunosuppressive effect of corticotherapy. Melatonin promotes a Th1-mediated response (Th1 cells produce far more interferon $\gamma$ (IFN- $\gamma$ ) and interleukin 2 (IL-2) than Th2 cells), thereby activating inflammatory pathways. However, melatonin can also stimulate Th2 cells which produce far more IL-4 and IL-10 than do the Th1 cells. Th2 cells stimulate antibody production via B lymphocytes and down regulate Th1 cells, and thus they may inhibit some Th1-mediated inflammatory responses [5-7].

Although melatonin is one of the least toxic substances known, its pharmacological effect on some autoimmune diseases such as rheumatoid arthritis and Crohn's disease at this point is controversial [8]. In 1997, Hong et al. [9] reported a patient who developed autoimmune hepatitis after beginning melatonin therapy for the treatment of insomnia. Recently, Fourman et al. [10] reported a case of autoimmune hepatitis that developed in a 50-year-old man after starting ramelteon, a melatonin agonist, for insomnia.
Here we present a patient with primary sclerosing cholangitis (PSC) with features of autoimmune hepatitis $(\mathrm{AlH})$ and associated with ulcerative colitis, who developed a severe elevation of liver enzymes following the use of melatonin in two separate courses.

The patient was a 25-year-old female with a history of autoimmune hepatitis diagnosed at the age 23 years in a local hospital and treated with corticosteroids and azathioprine for 2 years. She denied taking any other medications as well as supplements for depression including St John's wort, which has been known to cause serious interactions with some drugs. Because she experienced no response to the therapy she was referred to our hospital. On admission, she was asymptomatic. There was no history of risk factors for liver disease such as malnutrition, prior blood transfusion or surgical procedures, alcohol or drug abuse, or contact with high-risk individuals. Her family history was noncontributory, and her physical examination was unremarkable. Gynaecological and neurological examination also revealed no abnormalities. Laboratory tests are presented in Table I. The immunological profile was positive for anti-nuclear antibody and anti-smooth muscle antibody at titres of $1: 160$ and $1: 320$, respectively, and seronegative for anti-mitochondrial antibody, anti-liver/ kidney microsomal antibody, and perinuclear anti-neutrophil cytoplasmic antibody (done by indirect immunofluorescence). Results of serologic tests for hepatitis $A, B$, and $C$ viruses and for antibodies to cytomegalovirus (CMV) and Epstein-Barr viruses were negative. The result of polymerase chain reaction (PCR) testing for HCV-RNA was also negative. Normal values were obtained for the following parameters: $\alpha 1$-antitrypsin, ceruloplasmin, ferritin, albumin, triglycerides, and glucose. 
Table I. Clinical and laboratory findings at the time of admission

\begin{tabular}{lc} 
Parameter & Results \\
\hline Sex & Female \\
\hline Age [year] & 25 \\
\hline BMI $\left[\mathrm{kg} / \mathrm{m}^{2}\right]$ & 24.3 \\
\hline Glucose (normal range: 3.9-5.5 mmol/l) & 5.4 \\
\hline ALT (normal< 40 UI/l) & 200 \\
\hline AST (normal < 40 UI/l) & 129 \\
\hline ALP (normal < 120 UI/l) & 309 \\
\hline GGT (normal < 55 IU/l) & 258 \\
\hline Bilirubin (normal range: $0.2-1 \mathrm{mg} / \mathrm{dl})$ & 1.1 \\
\hline Cholesterol (normal range: $150-200 \mathrm{mg} / \mathrm{dl})$ & 258 \\
\hline IgA (normal range: 0.70-4.0 g/l) & 1.23 \\
\hline IgG (normal range: $7.0-16.0 \mathrm{~g} / \mathrm{l})$ & 17.39 \\
\hline IgM (normal range: $0.40-2.30 \mathrm{~g} / \mathrm{l})$ & 3.07 \\
\hline
\end{tabular}

Magnetic resonance cholangiopancreatography (MRCP) showed focally narrowed common bile duct indicating PSC. Colonoscopy revealed evidence of active ulcerative colitis (UC) with diffuse mucosal inflammation and ulcerations in the colon from the rectum to the proximal colon (pancolitis). Microscopic examination of biopsy specimens showed mucosal ulcerations, infiltration of lamina propria with inflammatory cells and crypt abscesses.

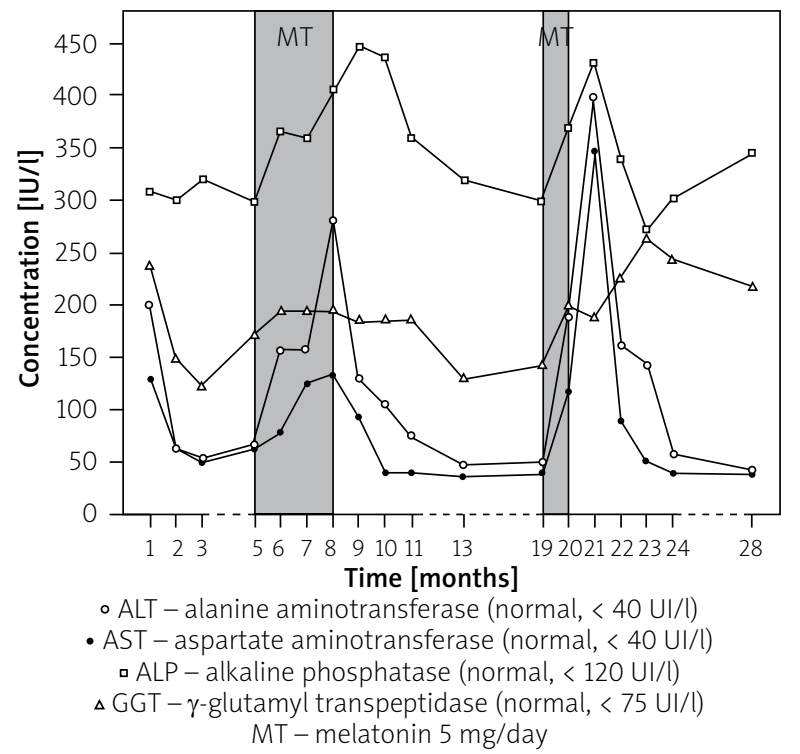

Figure 1. Biochemical events in relation to therapy
The liver biopsy revealed histological findings that resembled those found in the initial biopsy performed two years earlier in a local hospital. The findings included hepatocyte ballooning degeneration, mild inflammatory infiltration of portal zones primarily of lymphocytes with focal interface hepatitis, and mild portal fibrosis, but lymphocytes surrounding some of bile ducts were noted as a new feature. The patient was diagnosed with PSC with features of AlH associated with UC. Treatment was switched to mycophenolate mofetil (Celcept) $1000 \mathrm{mg}$ daily, mesalazine $3 \mathrm{~g}$ daily and ursodeoxycholic acid (UDCA) $15 \mathrm{mg} / \mathrm{kg}$ daily. However, because she did not tolerate mesalazine, she was left with mycophenolate mofetil and UDCA only. The patient was then discharged and was assessed at 4- to 6-week intervals in the outpatient clinic. Figure 1 presents biochemical events in relation to therapy. The patient's aminotransferase levels decreased gradually to normal within two months, whereas the cholestatic component remained abnormal. Approximately 4 months later, her serum liver enzyme levels abruptly rose. She was then admitted again. She remained asymptomatic. Complete blood count, serum bilirubin level, and prothrombin time were all normal. Serum testing for hepatitis B virus (HBV), hepatitis $C$ virus (HCV), and CMV viruses were negative. The macroscopic appearances of colonoscopy showed irregular mucosal surface without ulceration. The patient's drug history was revisited at that point, and we discovered that approximately 3 months before admission she had started to take melatonin $(5 \mathrm{mg})$ at bedtime for insomnia. We considered whether melatonin could be a potential cause of marked elevation of serum aminotransferase levels. The patient then stopped consuming melatonin while mycophenolate mofetil and UDCA were continually administered at unchanged doses. Her serum aminotransferase levels gradually declined and returned to pre-melatonin treatment levels during two months; however, cholestatic enzyme levels did not change significantly.

Following approximately seven months of successful management the patient again started by herself to take melatonin $5 \mathrm{mg}$ at bedtime to improve sleep propensity. After about three weeks of melatonin use, a routine liver enzymes test revealed a marked elevation of serum alanine aminotransferase (ALT) and aspartate aminotransferase (AST) levels from values near normal to approximately $10 \times$ upper limit of normal (ULN) and $8 \times$ ULN, respectively. Alkaline phosphatase (ALP) and $\gamma$-glutamyl transpeptidase (GGT) levels also rose; however, they were significantly elevated prior to melatonin administration (Figure 1). The patient was strongly recommended to withdraw melatonin and avoid it in the future. The existing daily doses of my- 
cophenolate motefil and UDCA were maintained. The aminotransferase levels decreased significantly 1 month after melatonin withdrawal, and they were minimally increased above or within the normal range during 18 months of follow-up. Currently, the AlH component of the AIH/PSC overlap is being successfully controlled with mycophenolate motefil, but an MRC, which was conducted as part of PSC follow-up, revealed evidence of extraand intrahepatic duct abnormalities consistent with PSC.

In our patient, the temporal association between administration of melatonin and elevation in liver enzymes observed over the two separate courses of this drug strongly suggest that melatonin caused the exacerbation of biochemical flares. The flare regression to melatonin withdrawal additionally supports this suggestion. Melatonin is not known to be hepatotoxic; therefore, in our patient the increase in liver enzyme levels during melatonin therapy is unlikely to have represented hepatotoxicity, but it is consistent with an exacerbation of the underlying autoimmune liver disease.

There are only a few published cases of the temporal relationship between melatonin use and the development or aggravation of autoimmune disorders. To date, there is only one case report [8] in a patient who developed autoimmune hepatitis after beginning melatonin therapy for the treatment of insomnia. In addition, Maestroni et al. [11] and Calvo et al. [12] postulated the disease-promoting role of melatonin in rheumatoid arthritis and Crohn's disease, respectively, but no such cases have been reported. Our report is clinically important because many people use melatonin to help improve their sleep.

Because of the very few side effects, melatonin is available without prescription in some European countries, while in the United States melatonin is considered a dietary supplement. While there are many situations in which melatonin has not been shown to aggravate autoimmune diseases, we suggest that caution should be exercised before initiating melatonin therapy in patients who potentially have autoimmune liver disease. Even if melatonin can be used in those patients, one should remember this side effect, especially after repeated doses.

\section{Conflict of interest}

The authors declare no conflict of interest.

\section{References}

1. Hardeland R, Pandi-Perumal S, Cardinali D, et al. Melatonin. Int J Biochem Cell Biol 2006; 38: 313-6.

2. Carrillo-Vico A, Guerrero J, Lardone P, et al. A review of the multiple actions of melatonin on the immune system. Endocrine 2005; 27: 189-200.
3. Cutolo M, Maestroni G. The melatonin-cytokine connection in rheumatoid arthritis. Ann Rheum Dis 2005; 64: 1109-11.

4. Boga JA, Caballero B, Potes Y, et al. A potential of melatonin related to its role as an autophagy regulator: a review. J Pineal Res 2019; 66: e12534.

5. Szczepanik M. Melatonin and its influence on immune system. J Physiol Pharmacol 2007; 58 (Suppl 6): 115-24.

6. Majewska M, Zajac K, Zemelka M, et al. Influence of melatonin and its precursor L-tryptophan on Th1 dependent contact hypersensitivity. J Physiol Pharmacol 2007; 58 Suppl 6: 125-32.

7. Kidd P. Th1/Th2 balance: the hypothesis, its limitations, implications for health and disease. Altern Med Rev 2003; 8: 223-46.

8. Sanchez-Barcelo E, Mediavilla M, Tan D, et al. Clinical uses of melatonin: evaluation of human trials. Curr Med Chem 2010; 17: 2070-95.

9. Hong Y, Riegler J. Is melatonin associated with the development of autoimmune hepatitis? J Clin Gastroenterol 1997; 25: 376-8.

10. Fourman L, Meyer R. Autoimmune hepatitis in association with ramelton. J Clin Gastroenterol 2013; 47: 651-4.

11. Maestroni GJ, Cardinali DP, Esquifino AL, Pandi-Perumal SR. Does melatonin play a disease-promoting role in rheumatoid arthritis ? Neuroimmunol 2005; 158: 106-11.

12. Calvo JR, Guerro JM, Osuna C, et al. Melatonin triggers Crohn's disease symptoms. J Pineal Res 2002; 32: 277-8.

Received: 28.08.2016

Accepted: 27.02.2019 